\title{
CONTRIBUIÇÃO AO CONHECIMENTO DA DISTRIBUIÇÃO ESPACIAL DE PAEPALANTHUS POLYANTHUS (BONG.) KUNTH (ERIOCAULACEAE) EM ÁREAS DE BAIXADA ÚMIDA DE DUNAS
}

Recebido em 23.03.95. Aceito em 08.01.96.

\author{
Tânia Tarabini Castellani' \\ Silvana Vieira ${ }^{1}$ \\ Karla Zanenga Scherer ${ }^{1}$
}

RESUMO: (Contribuição ao conhecimento da distribuição espacial de Paepalanthus polyanthus (Bong.) Kunt (Eriocaulaceae) em áreas de baixada úmida de dunas). A distribuição espacial de Paepalanthus polyanthus foi avaliada em função da variação topográfica e da cobertura vegetal associada, em um trecho de baixada úmida de dunas na Praia da Joaquina, Florianópolis, SC. Foram delimitadas 4 transecções, ao longo das quais, quadrados de $0.5 \mathrm{~m}^{2}$ foram dispostos de maneira contínua. A densidade de $P$. polyanthus e a porcentagem de cobertura vegetal foram determinadas para cada quadrado amostral, registrando-se também, as espécies de maior cobertura. Em todas as transecções, $P$. polyanthus apresentou um padrão de distribuição agregado (Índice de dispersão de Morisita Id $>1, P<0.001$ ), estando seus indivíduos mais concentrados em áreas intermediárias da microrrelevo. $P$. polyanthus não ocorreu nos trechos mais elevados de dunas, onde, também, a cobertura vegetal era mais baixa. Nas bordas de lago e em áreas alagáveis, sua densidade é reduzida ou mesmo não ocorre. A limitação de $P$. polyanthus nestes trechos é discutida com base em aspectos de sua dinâmica populacional, uma vez que $P$. polyanthus apresenta elevada taxa de mortalidade após alagamentos e, em áreas mais elevadas, registra-se baixa emergência e sobrevivência de plântulas, principalmente em períodos de menor pluviosidade. Uma provável associação negativa com Ischaemum minus parece ocorrer, podendo ser explicada por eventuais alagamentos ou por interferência da densa cobertura desta gramínea para a germinação e crescimento de $P$. polyanthus.

Palavras-chave: Paepalanthus polyanthus, Eriocaulaceae, distribuição espacial, baixada úmida de dunas.

\begin{abstract}
Contribution to the knowledge of the distribution of Paepalanthus polyanthus (bong.) Kunth (Eriocaulaceae) in areas of dunes). The distribution Pattern of Paepalanthus polyanthus was surveved in relation to topography variation and associated vegetation cover in a sand dune slack. Praia da Joaquina, Florianópolis, State of Santa Catarina, Brazil. Quadrats of 0.5 $\mathrm{m}^{2}$ were settled continuously along 4 line transects. The density of $P$. polyanthus, the percentage cover and the predominant species were determined in each quadrat. $P$. polyanthus showed a contagious pattern of distribution (Dispersion index of Morisita $I d>1, P<0.001$ ) in all transects. The individual were concentrated in intermediary areas of the microrelief. $P$. polyanthus was not
\end{abstract}

\footnotetext{
${ }^{1}$ Departamento de Ecologia e Zoologia. Centro de Ciências Biológicas. Universidade Federal de Santa Catarina. Caixa Postal 476, Florianópolis, SC. CEP 88040-960.
} 
observed at elevated sand dune areas, also characterized by lower percentage cover. At lake shores and flooded sites its density was low or the species absent. The factors limiting the distribution of $P$. polyanthus are discussed on aspects of its population dynamics. $P$. polyanthus exhibits high mortality after inundation and in higher sand dune areas lower emergence and seedling survival are observed, mainly in periods of low pluviosity. A negative association with Ischaemum minus is suggested and possibly explained by eventual floods or by interference of the dense cover of this grass species on germination and growth of $P$. polyanthus.

Key Words: Paepalanthus polyanthus, Eriocaulaceae, Spatial distribution pattern, sand dune slack.

\section{Introdução}

Estudos que enfocam a distribuição espacial de populações de plantas em ambientes de dunas mostram como fatores determinantes de distribuição: a) a ação de marés altas e a salinidade do solo; b) a concentração de nutrientes; c) a distância do lençol freático que, em parte, determina a freqüência de alagamentos e a umidade do solo; d) a mobilidade do substrato; e) a interferência da cobertura vegetal e, f) o favorecimento por espécie vegetal associada (Pfadenhauer 1978, Costa et al. 1988a,b, Costa \& Seeliger 1990, Mendonça \& Castellani 1993). Todos estes estudos têm uma abordagem demográfica, analisando a distribuição espacial também como resultado de processos de nascimento e morte de indivíduos de uma população.

Paepalanthus polyanthus é uma espécie monocárpica (Castellani 1990), herbácea rosetada que, no Estado de Santa Catarina, ocorre nos campos do planalto e nos campos arenosos do litoral (Moldenke \& Smith 1976).

Nos campos litorâneos, a distribuição dos indivíduos desta espécie vem sendo descrita como agrupada, Moldenke \& Smith (1976) referem-se a P. polyanthus como de dispersão descontínua, formando, em alguns trechos, densos agrupamentos. Locatelli (1988) descreve um padrão agregado para esta espécie, baseando-se no mapeamento de indivíduos em 3 parcelas distribuídas aleatoriamente em um trecho de baixada úmida de dunas. A autora mostra a ocorrência de um agrupamento mais denso ao redor de uma depressão, que supõe ter sido um lago temporário.

Informações sobre a dinâmica populacional desta espécie mostram diferentes taxas de recrutamento e de mortalidade de plântulas e adultos em áreas distintas quanto ao microrrelevo, que condicionam diferentes condições de umidade do solo, de afloramento do lençol freático, mobilidade de substrato e cobertura vegetal (Scherer 1993, Castellani et al. 1995). As informações sobre a demografia desta população estimularam e subsidiaram o presente estudo, que teve como objetivo descrever a distribuição espacial de Paepalanthus polyanthus, em relação a variações topográficas e de cobertura vegetal, em um trecho de baixada úmida de dunas.

\section{Material e métodos}

Este estudo foi realizado nas dunas da Praia da Joaquina (Florianópolis, SC), em um trecho de baixada úmida, distante cerca de 500 metros do mar (Figura 1). Nesta 


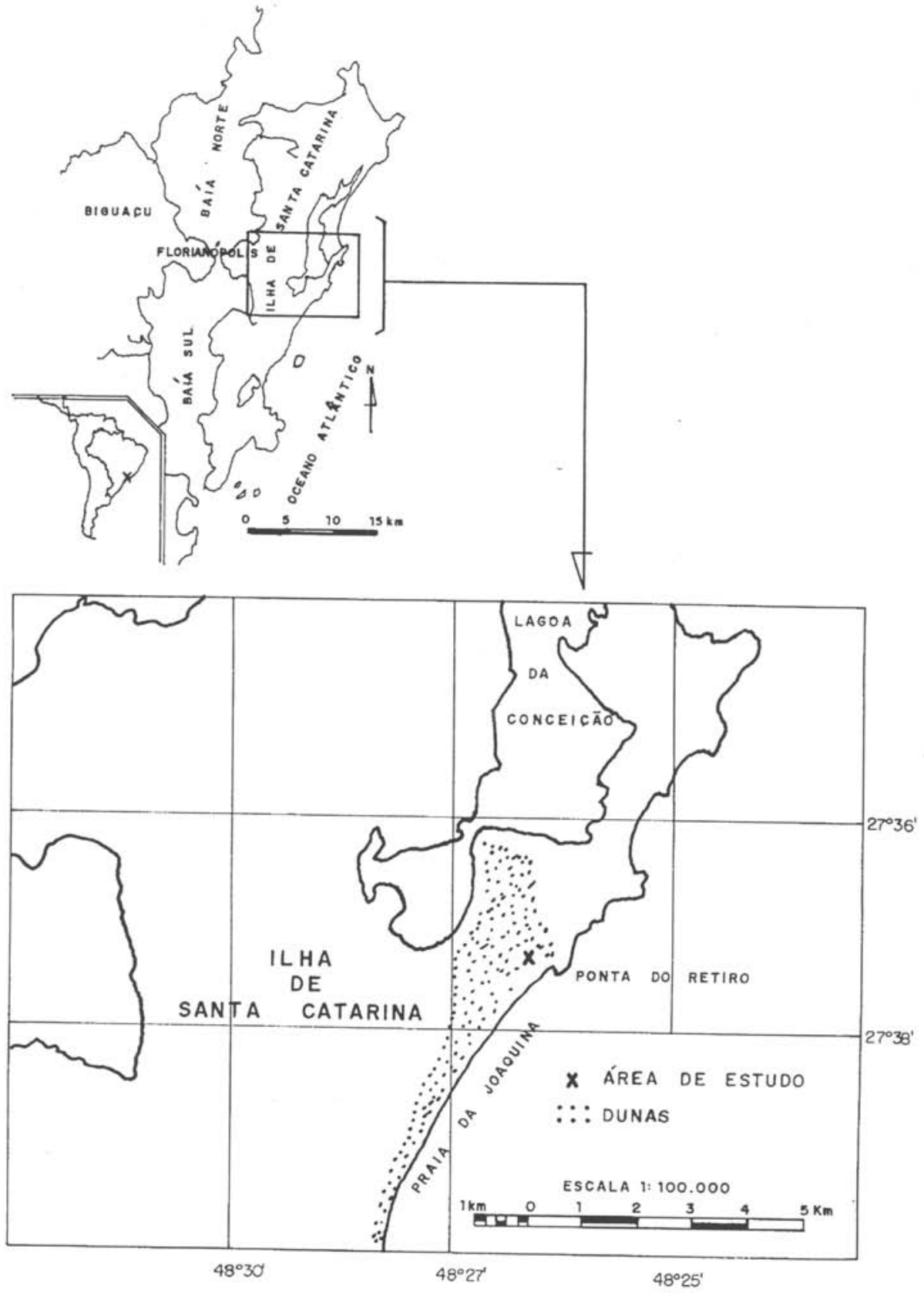

Figura 1. Localização da área de estudo nas dunas da Praia de Joaquina, Ilha de Santa Catarina, SC, Brasil. 
baixada encontram-se pequenos lagos permanentes, lagos temporários, depressões úmidas e alagáveis, entremeados com elevações de dunas de pequeno porte.

Foram feitas 4 transecções paralelas ao mar, dispostas aleatoriamente, em um

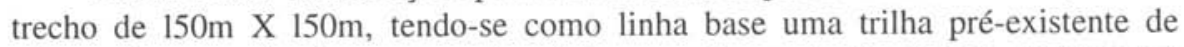
orientação perpendicular ao mar. A cada transecção, quadrados de $0.5 \mathrm{~m}^{2}(0.71 \mathrm{~cm} \mathrm{x}$ $0.71 \mathrm{~cm}$ ) foram amostrados de maneira contínua, sendo a amostragem interrompida quando da ocorrência de lagos. Um total de 72, 67, 78 e 24 quadrados foram delimitados nas transecções de 1 a 4, respectivamente, sendo a quarta transecção a de menor extensão devido à interrupção da amostragem por longo período chuvoso. A cada quadrado, determinou-se o número de plantas de Paepalanthus polyanthus presentes, a porcentagem de cobertura vegetal e as espécies predominantes. Para a estimativa de cobertura vegetal, cada quadrado foi subdividido em 4 partes, avaliando-se, visualmente, a porcentagem de área coberta por vegetação. A topografia das transecções foi feita pelo método de balizas descrito em Slingsby \& Cook (1986), considerando-se, como cota zero, as bordas dos lagos. Foi calculado o índice de dispersão de Morisita (Id) (Krebs 1989) para descrever o padrão de distribuicão espacial das plantas em cada transeccão.

\section{Resultados}

Com base no tamanho de quadrado amostral empregado Paepalanthus polyanthus apresentou uma distribuição agregada $(\mathrm{Id}>1, \mathrm{P}<0,001)$ em todas as transecções, estando a maioria dos indivíduos situados em trechos intermediários do microrrelevo (Figuras 2, 3, 4 e 5). A amplitude de variação altitudinal amostrada pelas 4 transecções é sintetizada na figura 6a. Nesta, verifica-se que, das 2017 plantas registradas no total de 241 quadrados, nenhuma ocorreu em trechos de duna com altitudes acima de $70 \mathrm{~cm}$, sendo baixa sua ocorrência em áreas de depressão. A maior densidade acorreu em trechos entre 10 e $50 \mathrm{~cm}$ de altitude, em relação à cota zero empregada.

Na transeccão $1,83 \%$ das plantas situava-se entre 10 e $30 \mathrm{~cm}$ de altitude (Figura 6b). P. polyanthus não ocorreu nos trechos mais elevados do perfil, nas bordas de lago, na baixada úmida e na baixada alagada (Figura 2). Neste perfil, como nos demais, a porcentagem de cobertura vegetal e as espécies predominantes variaram com a mudança altitudinal. Nos trechos mais elevados (A) a porcentagem de cobertura vegetal era baixa (até 20\%), adensando-se, em alguns locais, devido a Stylosanthes viscosa e Eupatorium casarettoi. Na baixada úmida (B) e bordas de lago (C) ocorreu elevada cobertura vegetal ( 80 a 100\%), com predominância de Ischaemum minus. Na baixada alagada (D), a predominância foi de Panicum parvifolium, que encontrava-se com a folhagem predominantemente morta. Em (E), observou-se elevada densidade de indivíduos de $P$. polyanthus, na maioria de pequeno porte, em área com predomínio de Andropogon selloanus e Stylosanthes viscosa.

Na transecção 2, Paepalanthus polyanthus mostrou uma distribuição mais ampla em relação a altitude (Figura 3 e $6 \mathrm{c}$ ), mantendo-se, porém, ausente em determinados trechos. Nas áreas mais elevadas do microrrelevo (A), P. polyanthus não 


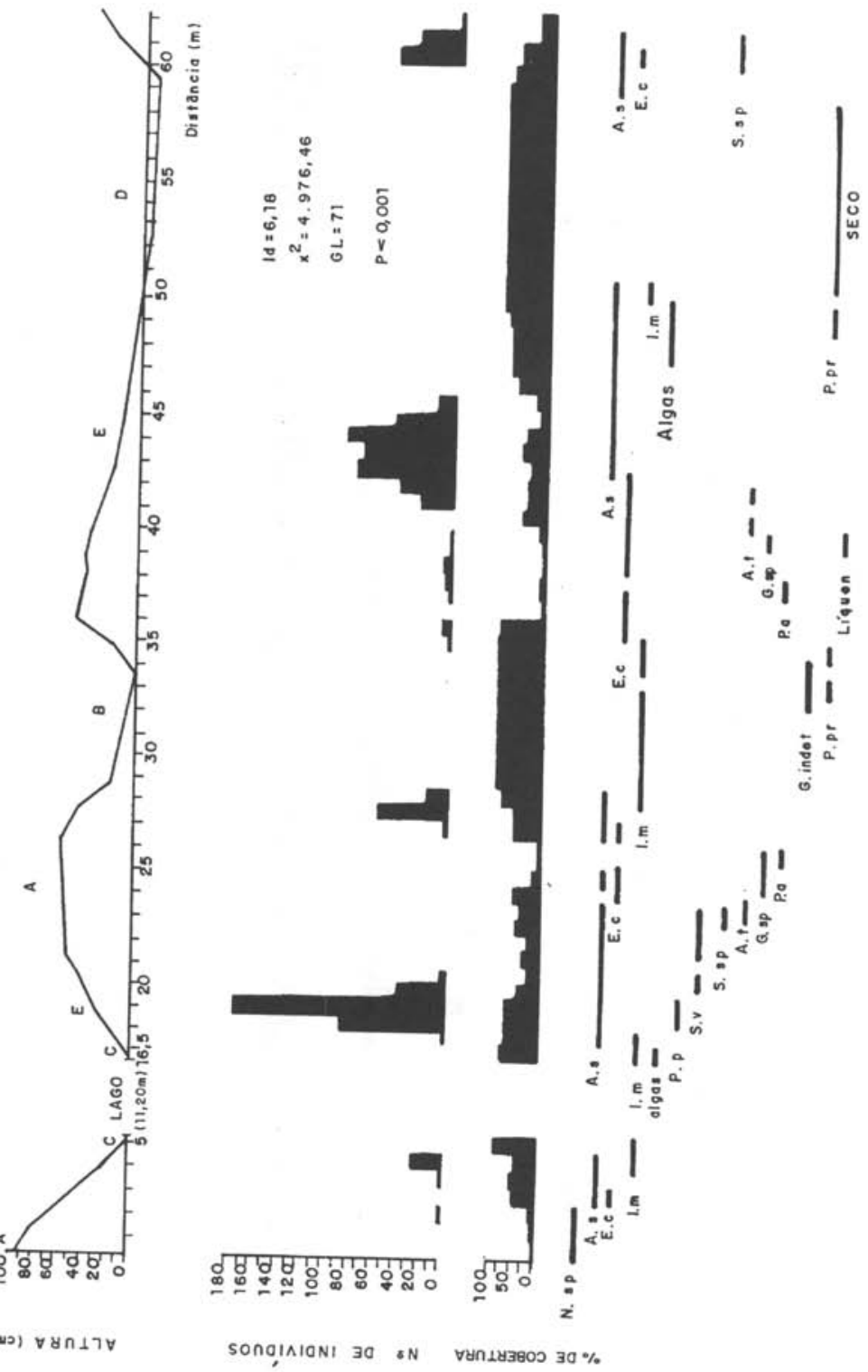

Figura 2. Perfil topográfico, número de indivíduos de Paepalanthus polyanthus, porcentagem de cobertura vegetal e espécies predominantes, em quadrados contíguos de $0.5 \mathrm{~m}^{2}$, ao longo da transecção 1 . As letras acima do perfil assinalam trechos discutidos no texto. Id- Índice de dispersão de Morisita.

N. sp.- Noticastrum sp., A.s- Andropogon selloanus, E.c- Eupatorium casarettoi, I.m- Ischaemum minus, P.p-Paepalanthus polyanthus, S.v-Stylosanthes viscosa, S.sp-Schizachyrium sp., A.t-Androtrichum trigynum, G.sp- Gymnopogon sp., P.a- Paspalum arenarium, G.indet.- Gramineae indeterminada, P.pr- Panicum parvifolium. 


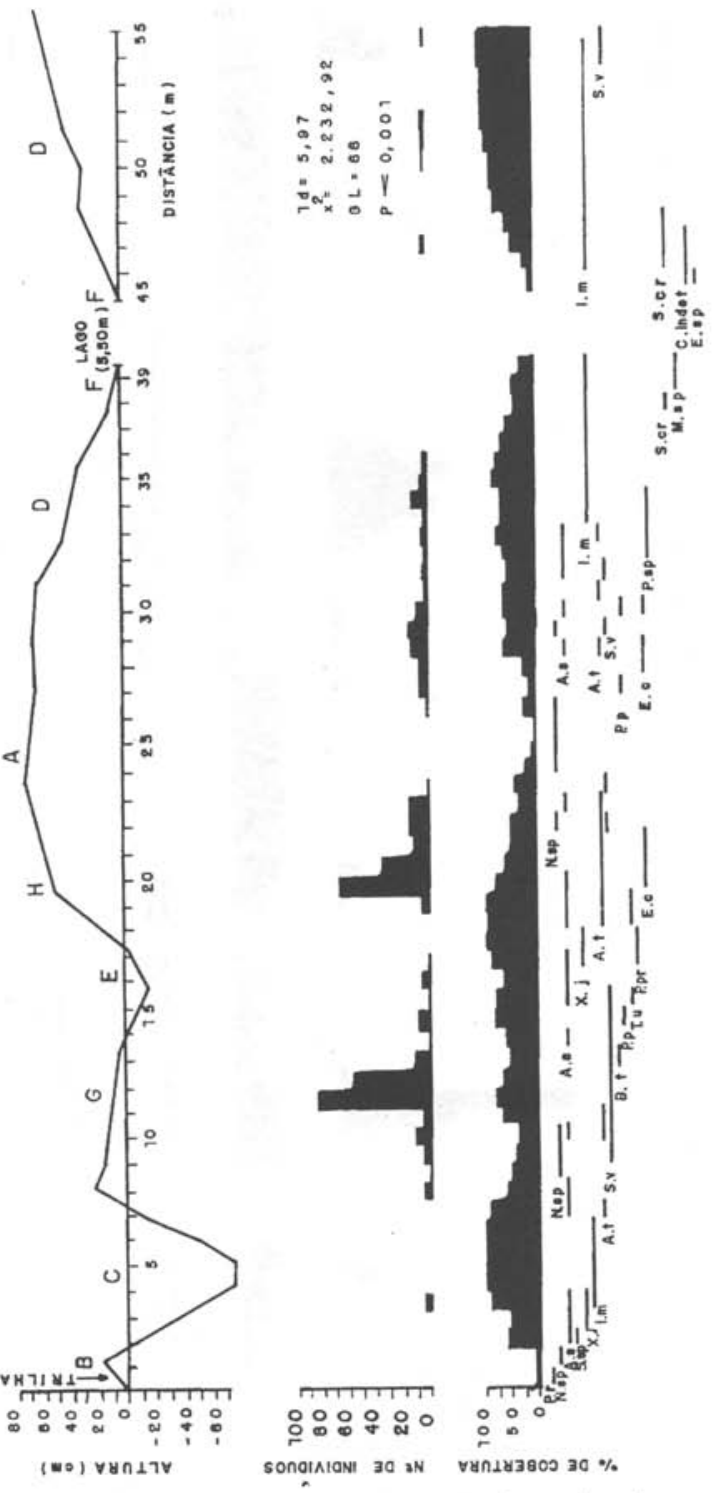

Figura 3. Perfil topográfico, número de indivíduos de Paepalanthus polyanthus, porcentagem de cobertura vegetal e espécies predominantes, em quadrados contínuos de $0,5 \mathrm{~m}^{2}$, ao longo da transecção 2 . As letras acima do perfil assinalam trechos discutidos no texto. Id- Índice de dispersão de Morisita.

P.r- Panicum racemosum, N.sp- Noticastrum sp., A.s- Andropogon selloanus, S.sp- Schizachyrium sp., X.j- Xyris jupicai, I.m- Ischaemum minus, A.t- Androtrichum trigynum. S.v- Stylosanthes viscosa, B.T- Baccharis trimera, P.p- Paepalanthus polyanthus, T.U- Tibouchina urvilleana, P.prpanicum parvifolium, E.c- Eupatorium casarettoi, P.sp- Paspalum sp., S.cr- Syngonanthus chrysanthus, M.sp- Mayaca sp., C. ident.- Cyperaceae indeterminada, E.sp- Elatine sp. 


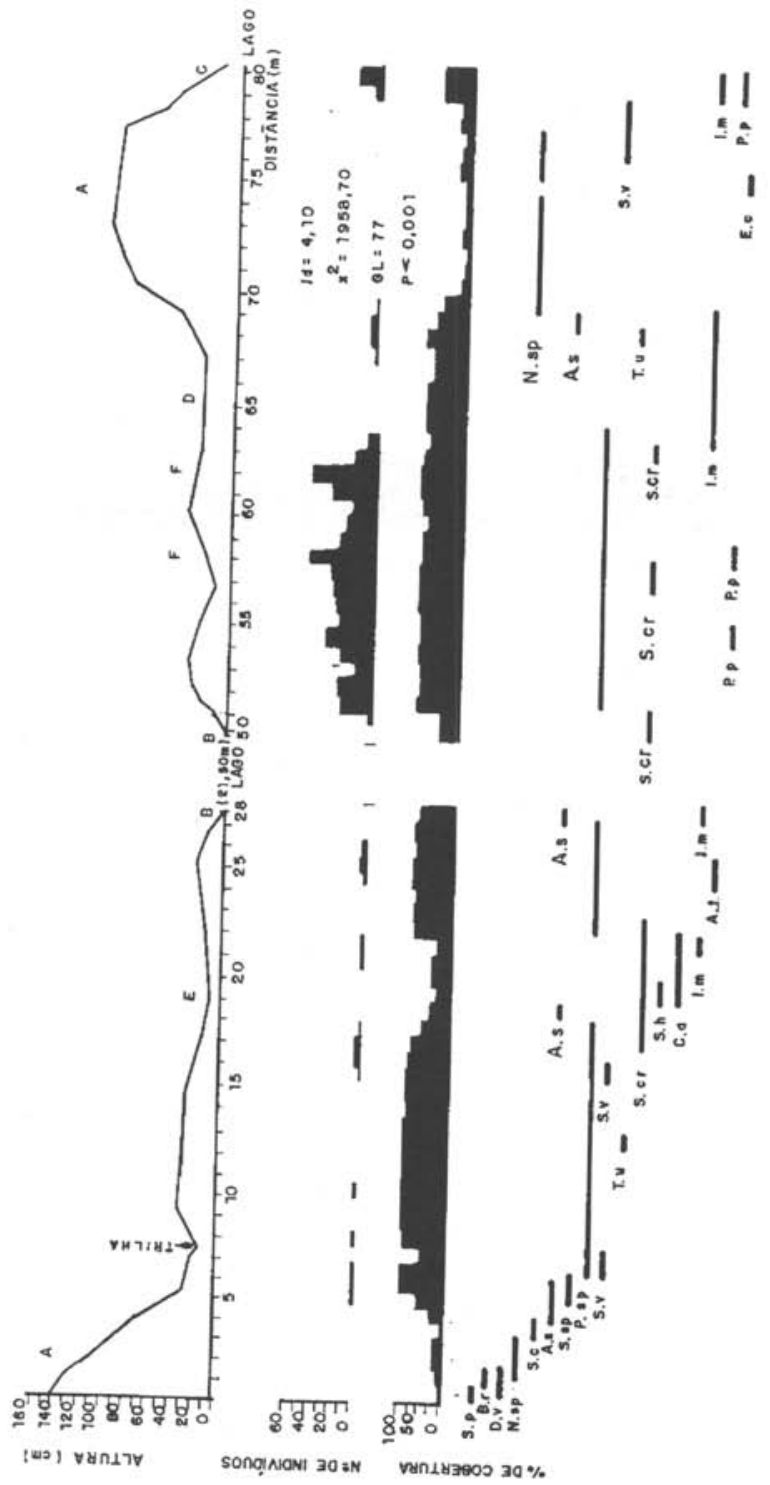

Figura 4. Perfil topográfico, número de indivíduos de Paepalanthus polyanthus, porcentagem de cobertura vegetal e espécies predominantes, em quadrados contíguos de $0,5 \mathrm{~m}^{2}$, al longo de transecção 3 . As letras acima do perfil assinalam trechos discutidos no texto. Id- Índice de dispersão de Morisita.

S.p- Senecio platensis, B.r- Baccharis radicans, D.v- Dodonaea viscosa, N.sp- Noticastrum sp., S.c- Sebastiania corniculata, A.s- Andropogon selloanus, S.sp- Schizachyrium sp., P.sp- Paspalum sp., S.v- Stylosanthes viscosa, T.u- Tibouchina urvilleana, S.cr- Syngonanthus chrysanthus, S.h- Scleria hirtella, C.a- Centella asiatica, I.m- Ischaemum minus, A.t- Androtrichum trigynum, P.p- Paepalanthus polyanthus, E.c- Eupatorium casarettoi. 


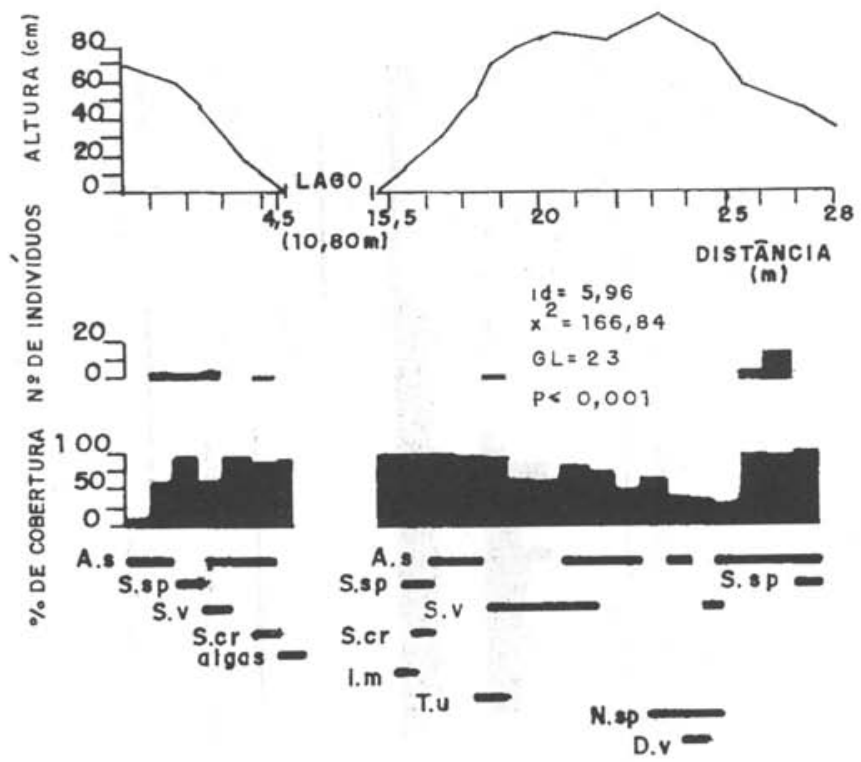

Figura 5.Perfil topográfico, número de indivíduos de Paepalanthus polyanthus, porcentagem de cobertura vegetal e espécies predominantes, em quadrados contíguos de $0,5 \mathrm{~m}^{2}$, ao longo de transecção 4 . As letras acima do perfil assinalam trechos discutidos no texto. Id- Índice de dispersão de Morisita.

S.p- Andropogon selloanus, S.sp- Schizachyrium sp., S.v- Stylosanthes viscosa, S.cr- Syngonanthus chrysanthus, L.m- Ischaemum minus, T.u- Tibouchina urvilleana, N.sp- Noticastrum sp., D.vDodonea viscosa.

ocorreu. Nestas áreas, a cobertura vegetal era baixa (5 a 20\%) e com predomínio de Noticastrum sp. Situação similar ocorreu no início da transecção (B), em um trecho de menor altitude, próximo a uma trilha. Este trecho era contínuo a uma grande depressão úmida (C), dominada por $I$. minus, com ausência de $P$. polyanthus. P. polyanthus ocorreu associada a esta gramínea apenas em alguns quadrados da baixada úmida (D). A espécie em estudo não ocorreu em parte da depressão (E), onde predominavam Andropogon selloanus, Androtrichum trigynum, Panicum parvifolium, Tibouchina urvilleana e Xyris jupicai. e nas bordas da lago (F). A maior densidade de plantas ocorreu em (G) e (H), áreas com cerca de 10 e $50 \mathrm{~cm}$ de altitude. com predomínío de A. selloanus, A. trigynum e Stylosanthes viscosa ou Eupatorium casarettoi.

Na transeccão $3,81.74 \%$ das plantas de $P$. polyanthus situava-se em áreas entre 20 e $40 \mathrm{~cm}$ de altitude (Figura 6d). Nesta transecção, as áreas mais elevadas atingiram $1,10 \mathrm{~m}$ e $1,40 \mathrm{~m}$ (A), tendo baixa cobertura vegetal, predomínio de Noticastrum sp. e ausência de $P$. polyanthus (Figura 4). No primeiro lago (B), esta espécie só ocorreu a partir de $20 \mathrm{~cm}$ e $12 \mathrm{~cm}$ acima das bordas, tendo-se Ischaemum minus, Syngonanthus chrysanthus e Paspalum sp. como as predominantes destas bordas. Na borda do segundo lago (C), observou-se P. polyanthus junto com I. minus. Na baixada(D), esta 
gramínea foi predominante, com baixa ocorrência de $P$. polyanthus. Em (E) observase uma depressão com predomínio de Centella asiatica, Scleria hirtella e S. chrysanthus, onde também não se registrou $P$. polyanthus.

Paspalum sp. ocorreu de maneira expressiva na transecção ora associada a uma elevada densidade de $P$. polyanthus, ora não (Figura 4). Em (F) observou-se que os indivíduos de $P$. polyanthus, apesar de numerosos, estavam muito amarelados, em função da densa cobertura desta gramínea.

P. polyanthus teve baixa ocorrência na transeccão 4 (Figuras 5 e 6e). Andropogon selloanus, Noticastrum sp. e Stylosanthes viscosa predominaram nos trechos mais elevados e, nas bordas de lago, predominaram A. selloanus, I. minus e S. chrysanthus.

A Figura 7 retrata um trecho da área em estudo durante a floração de Paepalanthus polyanthus. A situação mostrada foi descrita em algumas das transecções, tendose a ocorrência de $P$. polyanthus limitada, abaixo, por uma depressão úmida densamente ocupada por I. minus e, acima, limitada por trechos mais elevados e de menor cobertura vegetal.

\section{Discussão}

A maior concentração de plantas de Paepalanthus polyanthus em áreas de altitudes intermediárias do microrrelevo pode ser explicada, em parte, por aspectos de sua dinâmica populacional, que mostram maiores riscos de estabelecimento em áreas mais elevadas. Assim como, em áreas sujeitas a freqüentes alagamentos (Scherer 1993, Castellani et al. 1995). A ausência ou baixa densidade de indivíduos nos trechos de maior altitude e de baixa cobertura vegetal, podem estar relacionados a baixa emergência e sobrevivência de plântulas que ocorrem em áreas mais elevadas e secas, principalmente em períodos de baixa pluviosidade (Scherer 1993, Castellani et al. 1995). Nestas áreas, a dispersão e o soterramento de capítulos é mais acentuado, em função da baixa cobertura e maior mobilidade do substrato, fatores estes que também reduzem o recrutamento de plantas. Buckley (1982) e Naum \& Lapierre (1986) citam que quanto menores as sementes de uma planta, menores as chances de suas plântulas emergirem até a superfície, caso consigam germinar soterradas. P. polyanthus apresenta sementes com cerca de $0.5 \mathrm{~mm}$ de diâmetro, podendo ser consideradas de pequeno tamanho. Naum \& Lapierre (1986) discutem também, que a ausência de cobertura vegetal pode causar dessecamento das sementes, reduzindo o recrutamento.

Apesar de ocorrer maior germinação de Paepalanthus polyanthus sob condições mais úmidas, a elevada mortalidade de plantas após alagamentos (Scherer 1993, Castellani et al. 1995), pode explicar, em parte, sua baixa ocorrência em bordas de lago e em baixadas e depressões alagáveis. Alagamentos subseqüentes tendem a gerar um forte declínio populacional, principalmente em áreas onde os indivíduos morrem sem se reproduzir. Estudos em ambientes de baixada úmida de dunas reforçam o papel que as oscilações no nível do lençol freático têm na mortalidade e na germinação de sementes, determinando assim, as variações tempo- 


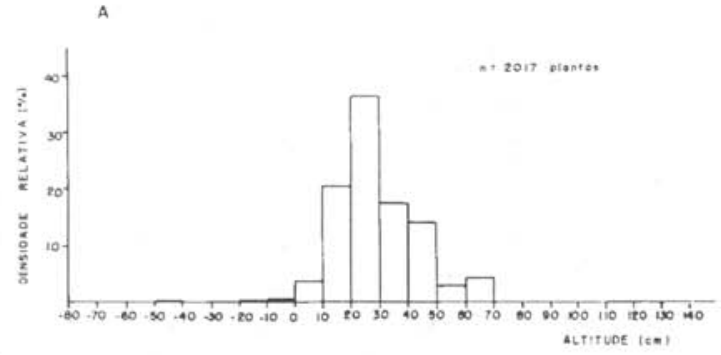

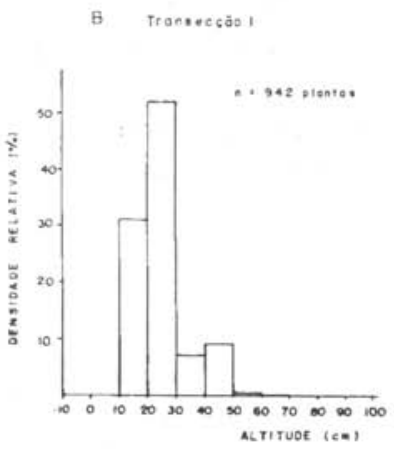

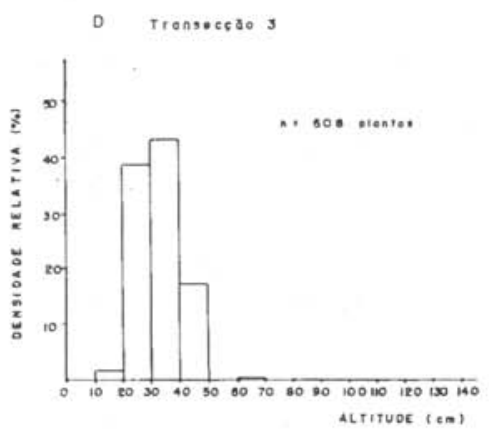

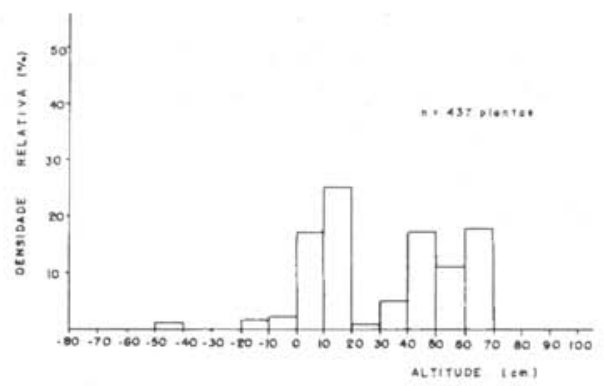

E

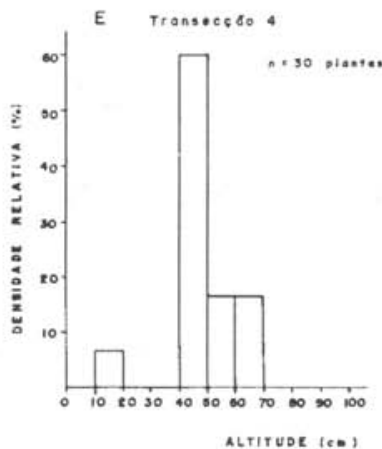

Figura 6. Densidade relativa de Paepalanthus polvanthus em áreas distintas quanto a altitude do microrrelevo, onde (a) apresenta os dados obtidos nas 4 transeç̧ões amostradas e (b). (c). (d) e (e) para as transeç̧ões 1. 2. 3 e 4 , respectivamente.

rais e espaciais das populações ao longo de perfis topográficos (Laan 1979, Schat 1983, Schat \& Scholten 1995).

A baixa ocorrência de plantas de $P$. polyanthus em áreas dominadas por Ischaemum minus pode ser devida a eventuais alagamentos ou à densa cobertura formada por esta gramínea, que poderia dificultar a germinação e o desenvolvimento das plântulas de $P$. polyanthus. A ausência de Drosera brevifolia em trechos de 


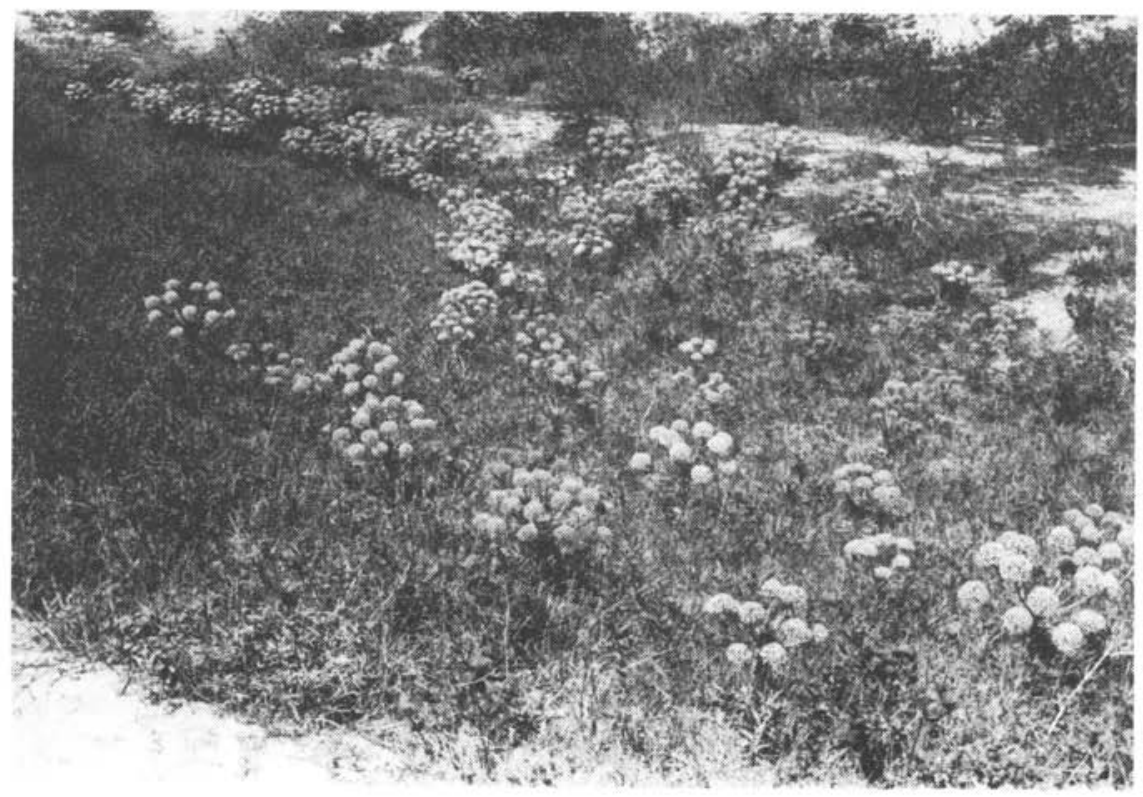

Figura 7.Distribuição de Paepulunthus polỵunthus ao redor de uma depressão úmida densamente coberta por lschaemun mimus. mostrando a baixa ocorrência de plantas nas áreas dominadas por esta gramineae e nas áreas mais elevadas e de menor cobertura vegetal. Dunas da Praia da Joaquina. Florianópolis. SC.

depressões úmidas com predomínio desta gramínea foi descrita por Mendonça \& Castellani (1993), onde se supôs que o denso tapete formado por I. minus poderia interferir no recrutamento de indivíduos de $D$. brevifolia. Muitas espécies de dunas mostram dependência de pequenas clareiras para estabelecimento inicial, havendo limitações ao recrutamento em áreas com densa cobertura vegetal (Meiiden \& WaalsKooi 1979, Boorman \& Fuller 1994, Klinkhamer \& De Jong 1988). Mesmo o baixo vigor de Paepalanthus polyanthus em áreas densamente cobertas por Paspalum sp. poderiam, também, sugerir uma interferência da cobertura vegetal ao pleno desenvolvimento desta planta.

A idéia de que os estudos sobre a distribuição espacial de plantas de dunas devem estar associados aos de dinâmica populacional para seu pleno entendimento (Ernst 1995) é defendida no presente trabalho. Apesar deste ser uma contribuição inicial ao conhecimento da distribuição espacial de $P$. polyanthus, verificamos que os limites de sua distribuição ao longo de perfis topográfícos, refletem os riscos de mortalidade e chances de germinação, principalmente os riscos relativos a fatores físicos do ambiente. As observações feitas neste estudo, levantam algumas questões especificas, como a da possível interferência da coberturta de $I$. minus, e mesmo de Paspalum sp. no estabelecimento de plantas desta população, sendo estes aspectos relevantes para investigações experimentais futuras. 


\section{Referências bibliográficas}

Boorman, L. A. \& Fuller. R. M. 1984. The comparative ecology of two sand dune biennials: Lactuca virosa L. and Cynoglossum officinale L. New Phytol. 69:609-629.

Buckley, R. C. 1982. Seed size and seedling establishment in tropical arid dunecrest plants. Biotropica 14(4): $314-315$.

Castellani, T. T.: 1990. Aspectos da ecologia reprodutiva de Paepalanthus polvanthus (Bong.) Kunth (Eriocaulacaeae) nas dunas da Praia da Joaquina, Ilha de Santa Catarina, SC. Anais do II Simpósio de Ecossistemas da Costa Sul e Sudeste Brasileira: estrutura, função e manejo. 1990. Águas de Lindóia: ACIESP. V.3. p.488-498.

Castellani, T. T.; Scherer, K. Z. \& Paula, G. S. 1995. Dinâmica populacional de Paepalanthus polyanthus (Bong.) Kunth (Eriocaulaceae) em baixada úmida de dunas: 5 anos de acompanhamento. Anais do III Congresso Latino-americano de Ecologia. Mérida. 1995. Mérida: Universidad de Los Andes. parte 13, p.6.

Costa. C. S. B. \& Seeliger, U., 1990. Quantitative phenology and horizontal distribution of the rhizomatous perennial herb Hydrocotyle bonariensis Lam. In coastal sand dunes. Vida Silv: Neotrop. 2(2): $36-42$.

Costa. C. S. B.: Seeliger. U. \& Cordazzo. C. V.: 1988a. Dinâmica populacional e distribuição horizontal de Androtrichum trigynum (Spreng.) Pfeiffer (Cyperaceae) em brejos e dunas costeiras do Rio Grande do Sul. Brasil. Acta Limnol. Brasil. 11: 913-842.

Costa. C. S. B.: Seeliger. U. \& Cordazzo. C. V. 1998b. Distribution and phenology of Andropogon arenarius Hackel on coastal dunes of Rio Grande do Sul. Brazil. Rev: Brasil. Biol. 48(3):527-536.

Ernst. W. H. O. 1985. Some considerations of and perspectives in coastal ecology. Vegetatio 62:533-545.

Klinkhamer. P. G. \& De Jong, T. J. 1988. The Importance of small scale disturbance for seedlinq stablishment in Cyrsium Vulgare and Cynoglossum Officinale. J. Ecol. 76:383-392.

Krebs. C. J. 1999. Ecological methodology: New York: Harper \& Row.

Laan. D. van der 1979. Spatial and temporal variation in the vegetation of dune slacks in relation to the ground water regime. Vegetatio 39:43-51.

Locatelli, L. M. 1988. Aspectos da dinâmica populacional de Paepalanthus polvanthus (Bong.) Kunth (Eriocaulaceae) em um trecho de dunas da Praia da Joaquina (Ilha de Santa Catarina). Florianópolis: Universidade Federal de Santa Catarina. Dissertação para conclusão de Curso de Graduação.

Meiiden, E. van der \& Waals-Kooi. R. E. Van der 1979. The population ecology of Senecio jacobaea in a sand dune system. J. Ecol. 67:131-153.

Mendonça. E. N. \& Castellani. T. T. 1993. Aspectos da ecologia populacional de Drosera brevifolia Pursh em um trecho de baixada úmida de dunas. Florianópolis, SC. Biotemas 6(1)31-49.

Moldenke, H. N. \& Smith. L. S. 1976. Eriocaulaceas, In Reitz, R. (ed.). Flora ilustrada Catarinense. Itajaí: Herbário Barbosa Rodrigues.

Naum, M. A. \& LaPierre, J. 1986. Effects of burial by sand on seed germination and seedling emergence of four species. Amer. J. Bot. 73(3):450-455.

Pfadenhauer, J. 1978. Contribuição ao conhecimento da vegetacão e de suas condições de crescimento nas dunas costeiras do Rio Grande do Sul. Brasil. Rev: Brasil. Biol. 38(4):827-836.

Schat, H. 1983. Germination ecology of some dune slack pioneers. Acta. Bot. Neerl. 32:203-212.

Schat, H. \& Scholten, M. 1985. Comparative population ecology of dune slack species: the relation between population stability and germination behaviour in brackish environments. Vegetatio 61:189-195.

Scherer, K. Z. 1993. Ecologia populacional de Paepalanthus polyanthus (Bong.) Kunth nas dunas da Praia da Joaquina. Itha de Santa Catarina. SC. Florianópolis: Universidade Federal de Santa Catarina. Dissertacão para conclusão de Curso de Graduação.

Slinasby, D. \& Cook, C. 1986. Practical ecology: London: MacMillan Education Ltd. 